\title{
The signaling axis of microRNA-31/interleukin-25 regulates Th1/Th17-mediated inflammation response in colitis
}

$\mathrm{T} \mathrm{Shi}^{1}, \mathrm{Y} \mathrm{Xie}^{1}, \mathrm{Y} \mathrm{Fu}^{1}, \mathrm{Q} \mathrm{Zhou}^{1}, \mathrm{Z} \mathrm{Ma}^{1}, \mathrm{~J} \mathrm{Ma}^{1}, \mathrm{Z} \mathrm{Huang}^{1}, \mathrm{~J} \mathrm{Zhang}^{1,2}$ and J Chen ${ }^{1,2}$

Interleukin-25 (IL-25) is an important regulatory cytokine that has a key role on mucosal immune tolerance during inflammation response. However, the molecular mechanism that regulates the colonic IL-25 expression in Crohn's disease (CD) remains unclear. In this study, IL-25 level was proved to decrease in 2,4,6-trinitrobenzene sulfonic acid (TNBS)-induced colitis mice and IL-10 knockout (KO) spontaneous colitis mice. An inverse correlation between IL-25 and miR-31 was discovered in the colons from model mice and CD patients. Furthermore, target validation analysis demonstrated that miR-31 directly regulated IL-25 expression by binding to its messenger RNA $3^{\prime}$-untranslated region. Changing colonic miR-31 level in the colitis mice could affect the mucosal IL-12/23-mediated Th1/Th17 pathway and lead to either amelioration or aggravation of colonic inflammation. In addition, the therapeutic effects of anti-miR-31 in TNBS-induced colitis were abolished by colonic treatment with IL-25 antibody or colonic down-expression of IL-25. Our findings demonstrated that IL-25 could be a crucial anti-inflammatory cytokine in TNBS-induced colitis and the signaling of miR-31 targeting IL-25 might be a possible mechanism that regulates IL-12/23-mediated Th1/Th17 inflammatory responses during colonic inflammation process. Restoring colonic IL-25 expression and blocking Th1/Th17 responses via intracolonic administration of miR-31 inhibitor may represent a promising approach for CD treatment.

\section{INTRODUCTION}

Crohn's disease $(\mathrm{CD})$ is a chronic relapsing inflammatory disorder that affects any part of the gastrointestinal tract. ${ }^{1,2}$ Although the precise etiology of CD is not well understood, several lines of evidence suggest that $\mathrm{CD}$ results from a combination of genetic susceptibility factors, environmental triggers, and mucosal immune imbalance. ${ }^{2-4}$ Among these pathogenic factors, cytokine imbalances are major contributors to mucosal inflammation. ${ }^{5,6}$ The increased expression of Th1 cytokines (IFN- $\gamma$ and TNF- $\alpha$ ) and Th17 cytokine (IL-17A) triggers the inflammatory responses in $\mathrm{CD},{ }^{7,8}$ meanwhile, there is a compelling evidence that abnormal expression of regulatory cytokines has a key role on mucosal immune tolerance during CD process. ${ }^{9}$

As a crucial regulatory cytokine, IL-25 (also known as IL17E) was initially considered to be a Th2 response-promoting factor in the control of immune-inflammatory responses. ${ }^{10-12}$
Mice deficient in IL-25 were shown to be highly susceptible to experimental autoimmune encephalomyelitis, which is a Th17 cell-driven autoimmune inflammatory disease of the central nervous system. ${ }^{13}$ In addition, the administration of recombinant IL-25 to experimental colitis or germ-free mice could significantly reduce Th1 or Th17 cells in the gut by suppressing the colonic expression of IL-12 or IL-23, which suggested that IL-25 was a counter-regulator of Th1/Th17-mediated inflammatory process. ${ }^{14,15}$ So far, the biological role of IL-25 has not been fully understood in colitis especially in CD. Although IL-25 production was monitored to be less in the gut for colonic inflammation patients when compared with normal controls, ${ }^{16}$ the molecular mechanism that regulates IL-25 expression in $\mathrm{CD}$ remains unclear.

MicroRNAs (miRNAs) are 22-nucleotide, evolutionarily conserved, non-coding RNAs that destabilize messenger RNAs and suppress translation. This mechanism occurs via the

${ }^{1}$ State Key Laboratory of Pharmaceutical Biotechnology, School of Life Sciences, Nanjing University, Nanjing, Jiangsu, China and ${ }^{2}$ State Key Laboratory of Analytical Chemistry for Life Sciences and Collaborative Innovation Center of Chemistry for Life Sciences, Nanjing University, Nanjing, Jiangsu, China. Correspondence: Z Huang (zhenhuang@nju.edu.cn) or J Zhang (jfzhang@nju.edu.cn) or J Chen (jnchen@nju.edu.cn) 
binding of miRNAs to the $3^{\prime}$-untranslated region ( $3^{\prime}$-UTR) of target mRNA molecules at the post-transcriptional level. ${ }^{17,18}$ Altered miRNA expression has been reported in the serum and mucosa of CD patients, suggesting that miRNA dysregulation might have a significant effect on the development of CD. ${ }^{19-22}$ Previous researches mostly investigated the regulation mechanism of these differentially expressed miRNAs on the pathogenic cytokines or transcription factors, such as TNF- $\alpha$, IL-12p40, SOCS3, MIP-2 $\alpha$, and CXCL12 $\beta .^{23-26}$ However, few studies have attempted to demonstrate the influence of miRNAs on colonic mucosal immune tolerance. An investigation is needed to determine whether aberrantly expressed miRNAs can influence the level of regulatory cytokines such as IL-25 during CD process.

In this study, we investigated the function of IL-25 in the experimental colitis and explored the potential molecular regulation of mucosal IL-25 expression during colonic inflammation. The regulation of IL-25 by miR-31 was respectively investigated in colonic epithelial cells (CECs), colonic macrophages (MФs), and two experimental colitis animal models (2,4,6-trinitrobenzene sulfonic acid (TNBS)induced colitis mice and IL-10 knockout (KO) spontaneous colitis mice). The inverse relationship between IL-25 and miR-31 was also investigated in CD patients. Moreover, the role of signaling axis of miR-31/IL-25 to regulate the colonic IL-12/23-mediated Th1/Th17 pathway was studied in TNBSinduced colitis via intracolonic administration of miR-31 precursor or inhibitor.

\section{RESULTS \\ IL-25 conferred protection against TNBS-induced and IL-10 KO colitis}

To investigate the function of IL-25 in TNBS-induced colitis and IL-10 KO colitis, recombinant IL-25 (rIL-25), or anti-IL-25 monoclonal antibody (IL-25 mAb) was administrated into colitic mice. Compared with the mice treated with TNBS alone, mice co-treated with TNBS and rIL-25 rapidly recovered the lost body weight, experienced low disease activity index (DAI), and had no significant signs of macroscopic colon inflammation (Figure 1a-c), whereas the lost body weight was recovered slowly for mice co-treated with TNBS, and IL-25 mAb and the mice exhibited high DAI and significant signs of macroscopic colon inflammation (Figure 1a-c). A similar result was found in IL-10 KO spontaneous colitis. IL-10 KO colitis mice treated with rIL-25 resulted in significantly decreased serum amyloid A (SAA) and slight colon inflammation, whereas IL-10 KO colitis mice given IL-25 mAb developed more severe colitis (Figure 1e,f).

The effects of rIL-25 or IL-25 mAb in two colitis models were further examined. rIL-25 treatment in colitis appeared to be associated with the reduction in colonic IL-12/IL-23 level, IFN$\gamma$ and IL-17A level (Th1/Th17 related cytokines), and T-bet and ROR $\gamma$ t expression (Th1/Th17 related transcription factors), whereas IL-25 mAb treatment in colitis might perform inverse effects (Supplementary Figure S1a-d). These results were confirmed by flow cytometry analysis, which indicated decreased colonic IFN- $\gamma^{+}$Th1 cells and IL-17A ${ }^{+}$Th17 cells proportion after rIL-25 treatment and increased IFN- $\gamma^{+}$Th1 cells, and IL-17A ${ }^{+}$Th17 cells proportion after IL-25 mAb treatment (Figure 1d,g). In addition, we also investigated whether IL-25 could regulate the immune homeostasis and barrier function in experimental colitis models. Enzyme-linked immunosorbent assay (ELISA) demonstrated that rIL-25 did not affect Th2-associated cytokines (IL-4, IL-5, and IL-13) in TNBS-induced and IL-10 KO colitic mice (Supplementary Figure S1e,g). The levels of Claudin-1, ZO-1, and Occludin (intestinal barrierassociated genes) were analyzed by quantitative real-time PCR (qRT-PCR), which demonstrated that rIL-25 could improve the intestinal barrier function in two colitic models (Supplementary Figure S1f,h).

To determine whether IL-25 could affect Th1/Th17 cell differentiation, naive $\mathrm{CD} 4^{+} \mathrm{T}$ cells were isolated and cultured with or without rIL-25 in Th1 or Th17 polarizing medium for 3 days. Flow cytometry analysis indicated that the presence of rIL-25 could not change the percentage of IFN $-\gamma^{+}$Th1 cells and IL-17A ${ }^{+}$Th17 cells (Supplementary Figure S2a,b).

\section{The inverse correlation analysis between miR-31 and IL-25 during colonic inflammation}

To determine which miRNAs may contribute to regulate IL-25 during colonic inflammation, we combined two algorithms: TargetScan and miRanda. With these bioinformatic approaches, 57 miRNAs potentially involved in regulating IL-25 were identified in Supplementary Table S1. As previously reported, ${ }^{26}$ the aberrantly expressed miRNAs of colon specimens in TNBS-induced and IL-10 KO colitis mice were shown in Supplementary Table S2. The intersection analysis between 57 miRNAs and the aberrantly expressed miRNAs in colitis models revealed that IL-25 might be targeted only by miR-31 during colonic inflammation (Figure 2a). The hybridization models between the $3^{\prime}$-UTR of mice or human IL-25 and miR-31 were shown in Figure 2b. There was perfect base pairing between the "seeds" (the core sequence that encompasses the first 2-8 bases of the mature miRNA) and cognate targets, and the "seeds" were conserved among species.

Furthermore, the time course experiments showed that the colonic IL-25 began to significantly decrease $24 \mathrm{~h}$ after TNBS challenge, whereas miR-31 in the colon significantly increased $12 \mathrm{~h}$ after treatment with TNBS (Figure 2c), suggesting the inverse correlation between miR-31 and IL-25 in TNBS-induced colitis models. The inverse correlation between miR-31 and IL-25 was also identified in the inflamed colons of IL-10 KO mice (Figure 2d). In addition, the results in Figure 2e indicated the impaired IL-25 production and the elevated miR-31 level in the inflamed sites of $\mathrm{CD}$ patients.

\section{In vitro validation of IL-25 as a target of miR-31}

Luciferase assays were performed to determine whether miR-31 could directly regulate IL-25. Overexpression of miR-31 impaired the activity of luciferase reporter containing the full-length $3^{\prime}$-UTR of wild-type mouse or human IL-25, whereas inhibition of miR-31 resulted in the increased reporter 

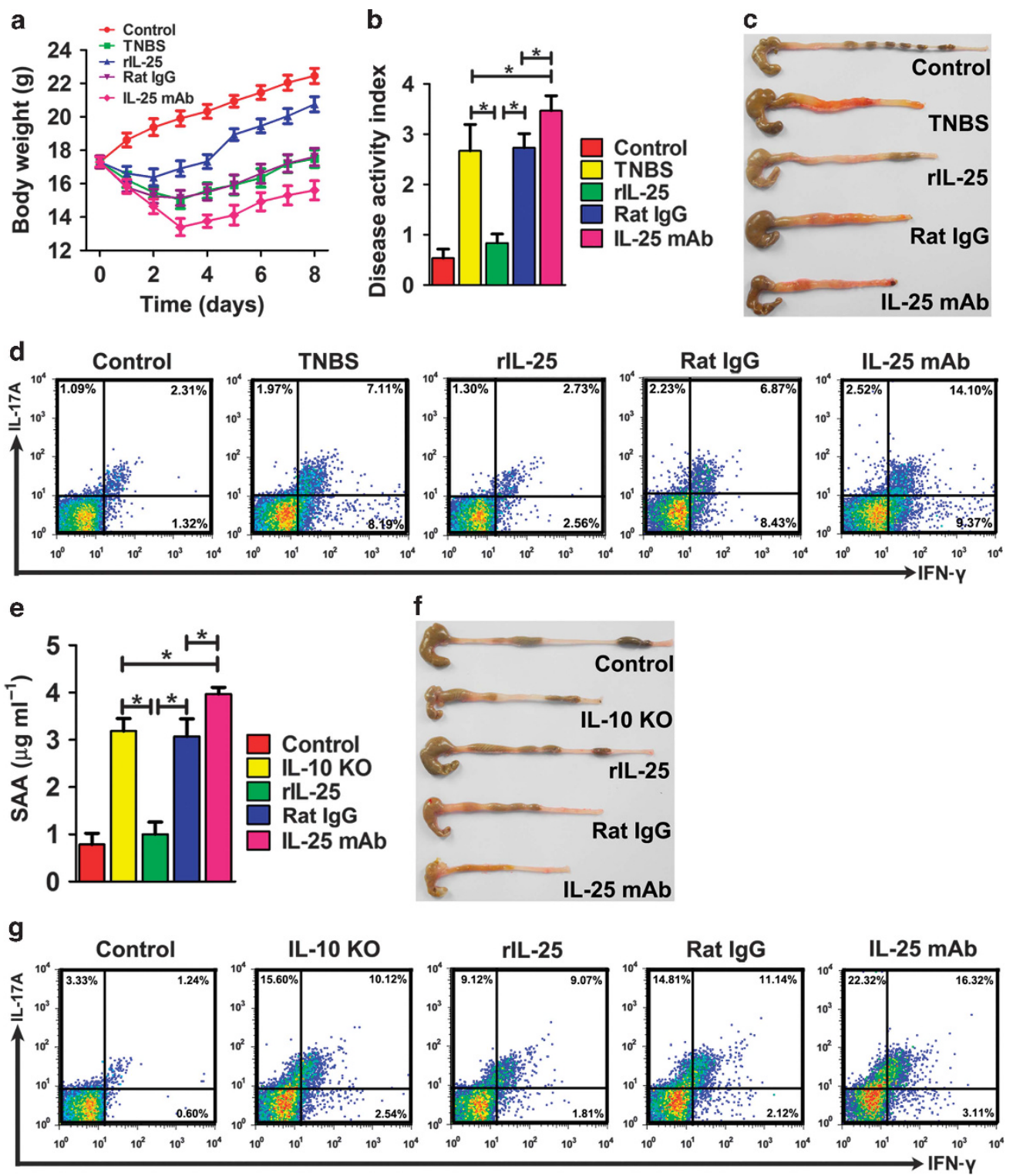

Figure 1 IL-25 conferred protection against 2,4,6-trinitrobenzene sulfonic acid (TNBS)-induced and IL-10 knockout (KO) colitis. Clinical evolution was examined based on body weight loss (a), DAI (b), and colon images (c) in TNBS-colitic mice after rlL-25 or IL-25 mAb treatment. Flow cytometry used to analyze the ratio of Th1/Th17 cells in colon mucosal CD4 ${ }^{+}$T cells from TNBS-colitic mice after rlL-25 or IL-25 mAb administration (d). The clinical effect of rlL-25 or IL-25 mAb treatment on IL-10 KO colitis was determined by SAA level (e) and colon images (f). Flow cytometry used to analyze the ratio of Th1/Th17 cells in colon mucosal CD4 ${ }^{+} \mathrm{T}$ cells from IL-10 KO colitic mice after rlL-25 or IL-25 mAb administration (g). The data are expressed as the mean \pm s.e.m. $n=9-11$ mice per group. ${ }^{*} P<0.05$.

activity compared with the control group (Figure 3a,b). Mutagenesis of the miR-31 binding sites abolished all miR-31mediated regulatory effects (Figure $\mathbf{3 a}, \mathbf{b}$ ). The results of qRT-PCR and ELISA in mouse primary cells or human colon cell lines also indicated the correlation between miR-31 and IL-25. Upregulation of miR-31 significantly reduced mRNA and protein levels of IL-25 in mouse CECs and colonic MФs or in human Caco-2 cells and THP-1 cells, whereas miR-31 inhibitors enhanced the levels of IL-25 transcript and protein in these cell types (Figure 3c-f and Supplementary Figure S3). The above results were further confirmed using western blotting analysis. As shown in Figure 3g,h, IL-25 protein expression was significantly decreased in cells treated with premiR-31 or enhanced in cells treated with anti-miR-31. These results suggested that miR-31 negatively regulated IL-25 expression.

\section{In vitro miR-31/IL-25 pathway regulated IL-12/23 production in antigen-presenting cells}

We further investigated the effect of miR-31/IL-25 axis on antigen-presenting cells (APCs) to explore the reason for reduced IL-12/IL-23 level in colon of colitis mice. Supernatants were collected from TNBS CECs after treated with pre/anti-miR-31 for $24 \mathrm{~h}$. Then the supernatants were added 
a
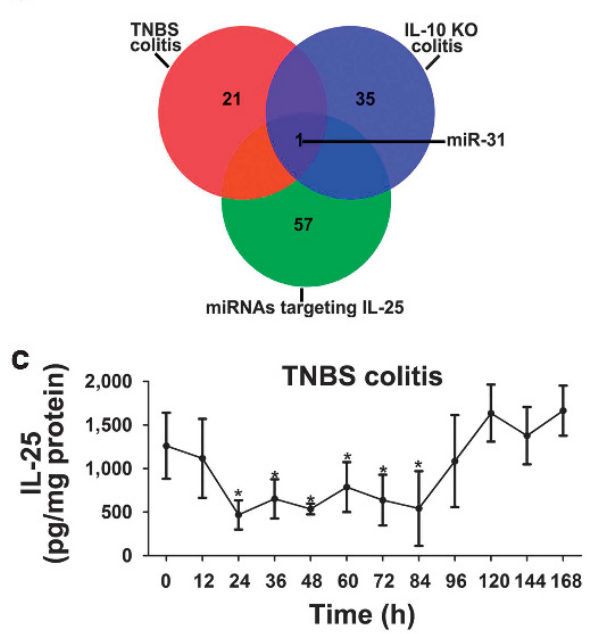

b
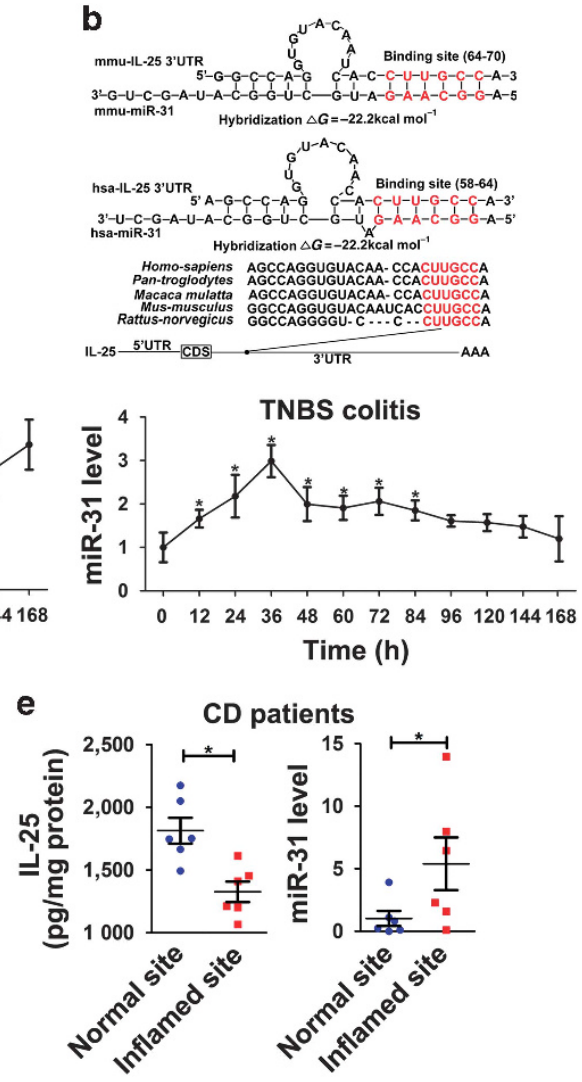

d

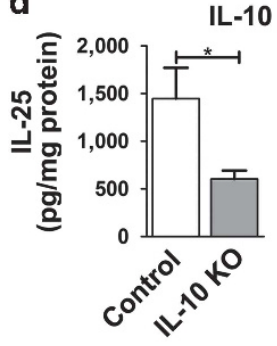

KO colitis

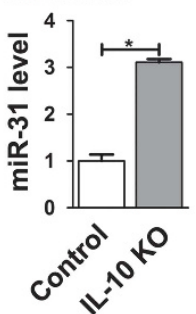

Figure 2 Correlation analysis between IL-25 and miR-31 in experimental colitis and Crohn's disease (CD) patients. Intersection analysis between microRNAs (miRNAs) targeting IL-25 and aberrantly expressed miRNAs in 2,4,6-trinitrobenzene sulfonic acid (TNBS)-induced/IL-10 knockout (KO) colitis models (a). Schematic description of the binding site between miR-31 and mouse/human IL-25 3'-untranslated region. The seed-recognizing sites were marked and the distances from the coding sequences to the binding sites were listed. The predicted free energy of each hybrid was indicated (b). IL-25 production detected by ELISA and miR-31 level performed by quantitative real-time PCR in the development of TNBS-induced colitis. MiR-31 level was indicated as relative expression compared with that of zero point (c). $n=9-11$ mice per group. IL-25 production and miR-31 level in IL-10 KO colitis (d). $n=9-11$ mice per group. IL-25 production and miR-31 level in six CD patients (e). The data are expressed as the mean \pm s.e.m. ${ }^{\star} P<0.05$.

to APCs, which were also isolated from TNBS-colitic colon. The IL-12/IL-23 level was significantly upregulated in APCs after treated with supernatant from pre-miR-31-transfected TNBS CECs, whereas the supernatant from anti-miR-31 treated CECs downregulated the expression of IL-12/23 in APCs (Figure 3i). In contrast, rIL-25 added into the supernatant from pre-miR31 treated CECs abrogated the upregulation of IL-12/23 by APCs (Figure 3i), whereas the addition of IL-25 mAb to the supernatant of anti-miR-31-treated CECs recovered the production of IL-12/23 in APCs (Figure 3i), suggesting that the signaling axis of miR-31/IL-25 might regulate IL-12/23 production through targeting APCs.

\section{Cellular localization of miR-31 and IL-25 in mouse and human colon}

To identify the cellular sources of miR-31 and IL-25 in colon, in situ hybridization/immunofluorescence was performed. In colon tissues of healthy mice and non-inflamed sites of $\mathrm{CD}$ patients, miR-31 was weakly expressed by CECs $\left(\mathrm{PCK}^{+}\right.$cells) and colonic МФs. In contrast, miR-31 was significantly increased in CECs and colonic MФs rather than in smooth muscle cells (SMCs) in the inflamed colon of TNBS colitis mice and CD patients. Moreover, abundant IL-25 expression was observed in CECs and colonic MФs of the normal colon tissues; however, few expression of IL-25 could be detected in CECs and colonic MФs of the colitic colons (Figure $\mathbf{4 a}$ and Supplementary Figure S4). As shown in Figure $\mathbf{4 b}$-d, the results from qRT-PCR and western blotting, performed in different colonic cell subsets, further confirmed that the inverse correlation between miR-31 and IL-25 existed in CECs and colonic MФs but not in SMCs. These results imply that IL-25 and miR-31 may be predominantly expressed in the identical cell types in the mouse/human colon but under opposing conditions.

\section{MiR-31 affected Th1/Th17 responses in TNBS-induced colitis after intracolonic administration of anti-miR-31}

In situ hybridization and immunofluorescence staining were first performed to determine whether intracolonic administration of anti-miR-31 could decrease miR-31 level and upregulate IL-25 production in colon tissues. Following intracolonic administration of miR-31 inhibitors for $72 \mathrm{~h}$, IL-25 level significantly increased in both CECs and colonic MФs of TNBS-colitic colons (Figure 5a). CECs and colonic 

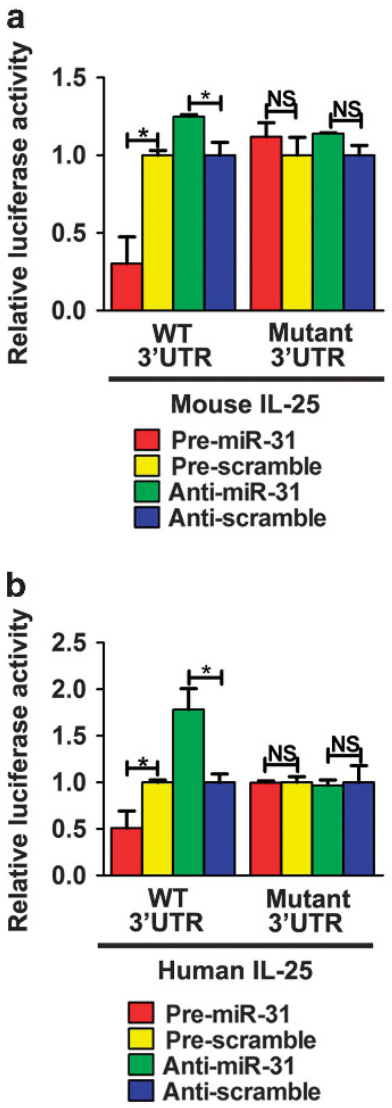

g
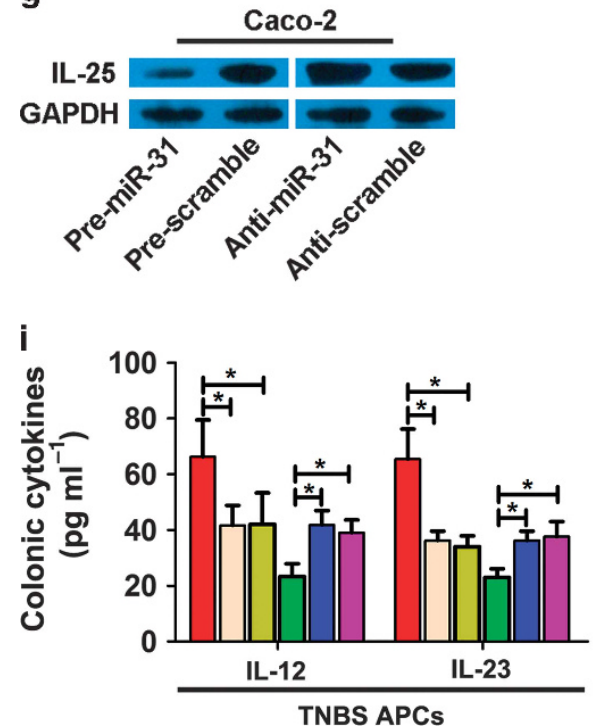

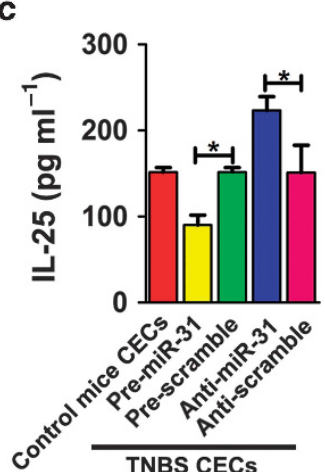

d

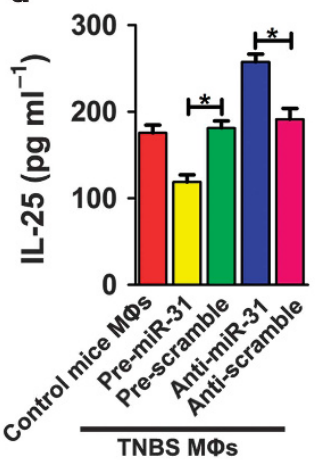

e

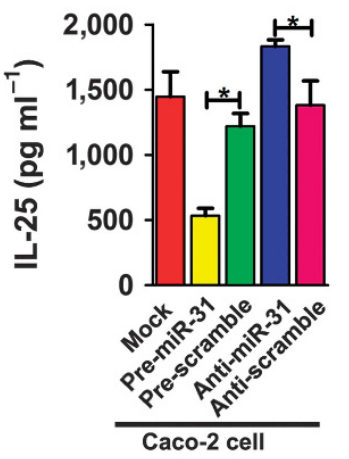

f

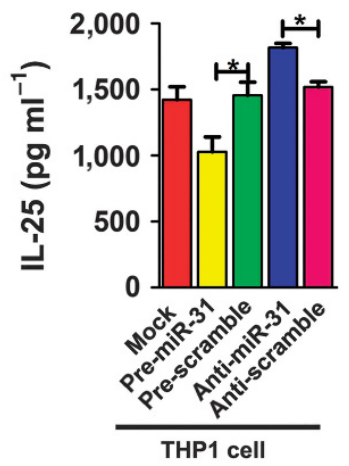

h

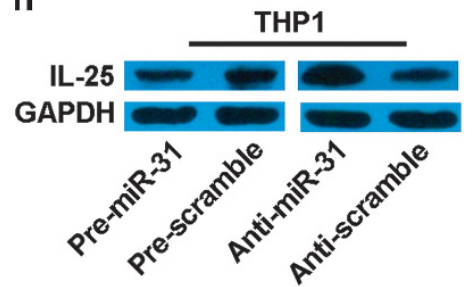

$\square$ Pre-miR-31

$\square$ Pre-scramble

$\square$ Pre-miR-31+rIL-25

$\square$ Anti-miR-31

$\square$ Anti-scramble

$\square$ Anti-miR-31+IL-25 mAb

Figure 3 Identification of miR-31 targeting IL-25 and biological effects of miR-31/IL-25 axis on antigen-presenting cells. The luciferase activity of a reporter containing the full-length $3^{\prime}$-untranslated region of mouse/human IL-25 were assayed after transfection of pre/anti-miR-31 or pre/anti-scramble into primary mouse colonic epithelial cells (CECs) (a) or Caco-2 cells (b). Meanwhile, the binding site mutant plasmid (mutant) was used. IL-25 concentration in supernatants from CECs (c), colonic MФs (d), Caco-2 cells (e), and THP-1 cells (f) were examined by ELISA after mock, pre/anti-miR-31, or pre/anti-scramble transfection. Cellular IL-25 protein expression was examined by western blotting after transfected with pre/anti-miR-31 or pre/antiscramble in Caco-2 (g) or THP-1 cells $(\mathbf{h})$. The western blotting is representative of three replicated experiments. The production of IL-12/IL-23 in antigenpresenting cells was detected after exposed to the supernatant of pre/anti-miR-31 treated 2,4,6-trinitrobenzene sulfonic acid CECs, or above supernatant added with rlL-25 (100 $\left.\mathrm{ng} \mathrm{ml}^{-1}\right)$ or IL-25 mAb $\left(2 \mu \mathrm{g} \mathrm{ml}^{-1}\right)$ (i). Five samples were analyzed per condition and all transfection experiments were performed in triplicate. The data are expressed as the mean \pm s.e.m. ${ }^{\star} P<0.05$. NS, no significant change. 


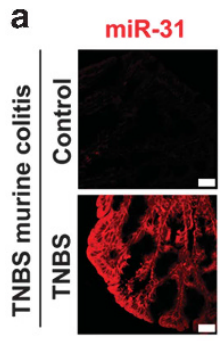

miR-31

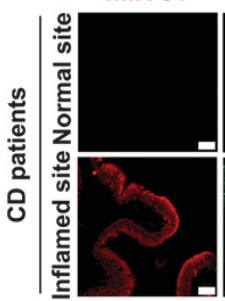

miR-31

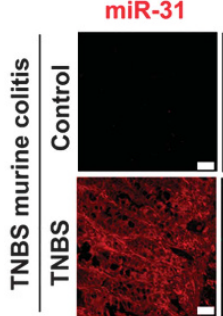

miR-31

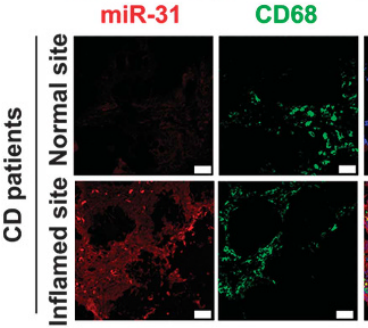

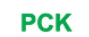

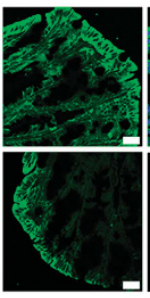

PCK

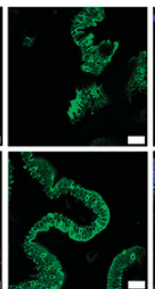

F $4 / 80$
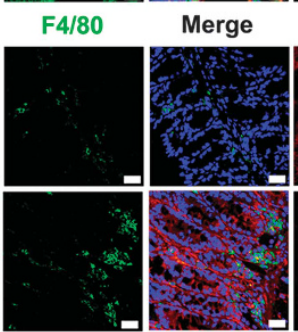

Merge

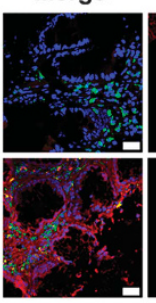

IL-25

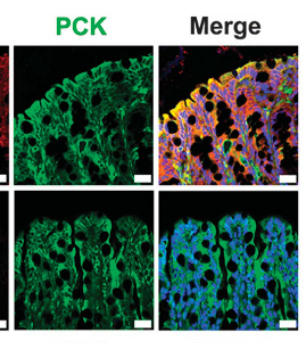

PCK

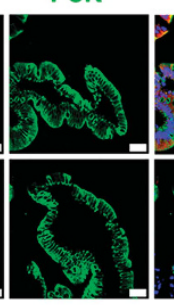

IL-25

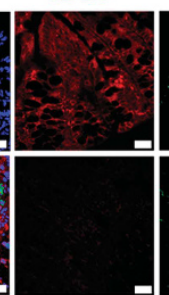

IL-25

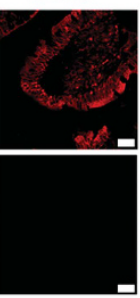

Merge

CD68

b
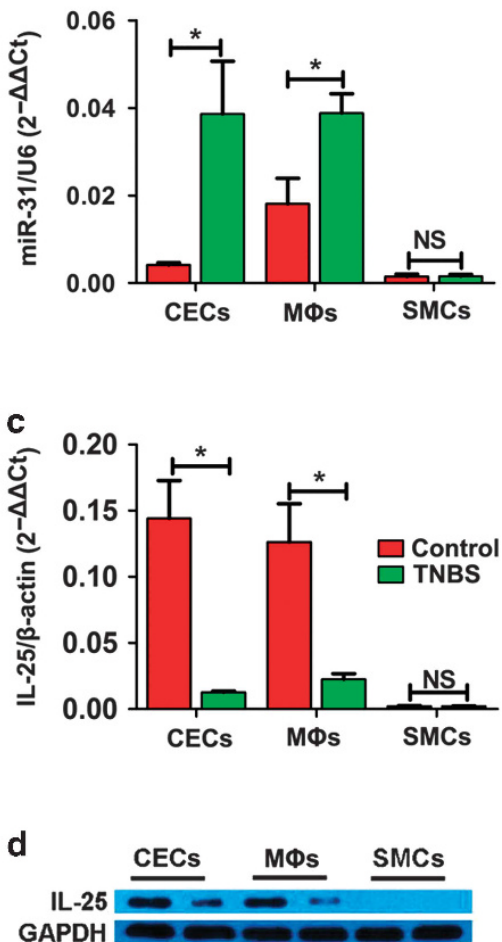<smiles></smiles>

Figure 4 Localization of miR-31 and IL-25 in 2,4,6-trinitrobenzene sulfonic acid (TNBS)-colitic colons and Crohn's disease (CD) patient colons. In situ hybridization for miR-31 was performed on colon sections from TNBS-colitic mice and CD patients (red, miR-31; green, PCK/F4/80/CD68; blue, DAPI nuclear staining), whereas IL-25 immunofluorescence staining was carried out to investigate its cellular localization (red, IL-25; green, PCK/F4/80/CD68; blue, DAPI nuclear staining) (a). Scale bar, $25 \mu \mathrm{m}$. One representative image of five repeats was shown. MiR-31 level (b), IL-25 mRNA level (c), and IL-25 protein level (d) were examined in different cell types isolated from TNBS-colitic colons. The data are expressed as the mean $\pm \mathrm{s}$. e.m. $n=9-11$ mice per group. NS, no significant change. ${ }^{\star} P<0.05$.

MФs were isolated $24 \mathrm{~h}$ after anti-miR-31 injection, and then significantly reduced miR-31 and upregulated IL-25 were examined in both CECs and colonic MФs by using qRT-PCR and western blotting analysis (Figure $\mathbf{5 b} \mathbf{b}-\mathbf{d}$ ).

To further investigate the effects of miR-31 on Th1/Th17 responses, APCs in the colon tissues were isolated from mice $24 \mathrm{~h}$ after anti-miR-31 treatment. ELISA demonstrated that IL-12/IL-23 expression from isolated APCs was significantly suppressed by anti-miR-31 (Figure 5e).

\section{Therapeutic effects of anti-miR-31 in murine colitis}

Anti-miR-31 treatment significantly decreased miR-31 level and promoted IL-25 expression in TNBS-colitic colons (Figure 6a,b) and the colons showed no significant signs of macroscopic inflammation following treatment with anti-miR31 (Figure 6c). Moreover, TNBS-treated mice given anti-miR31 rapidly recovered the lost body weight and exhibited low
DAI (Supplementary Figure S5a,b). Colonic myeloperoxidase (MPO) activity, which is correlated with mucosal neutrophil infiltration, was also reduced significantly (Supplementary Figure S5c). Notable improvements in histological characteristics were observed in the mice treated with antimiR-31 (Supplementary Figure S5d,e).

The therapeutic effects of anti-miR-31 were also evaluated in IL-10 KO spontaneous colitis. Repeatedly administration of anti-miR-31 in the IL-10 KO mice downregulated the colonic miR-31 level and promoted IL-25 expression (Figure 6e,f). SAA level and colonic MPO activity indicated that the anti-miR-31-treated IL-10 KO mice developed less severe colitis (Supplementary Figure S6a,b), and these results were confirmed by macroscopic observation (Figure 6g) and histological examination (Supplementary Figure S6c,d).

In addition, colonic levels of IL-12 and IL-23 were significantly reduced after treated with anti-miR-31 in the 
a

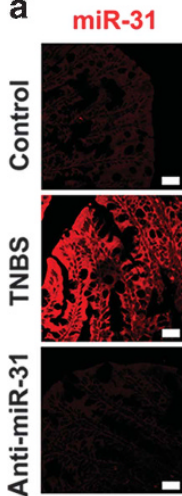

miR-31
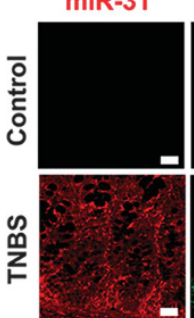

.
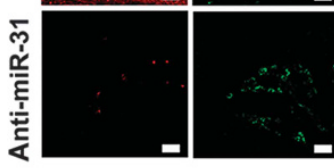

$\mathrm{F} 4 / 80$
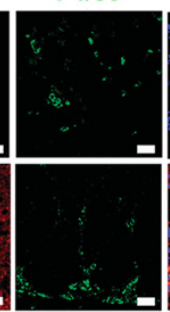

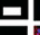
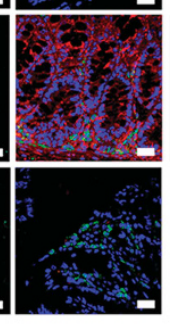

d
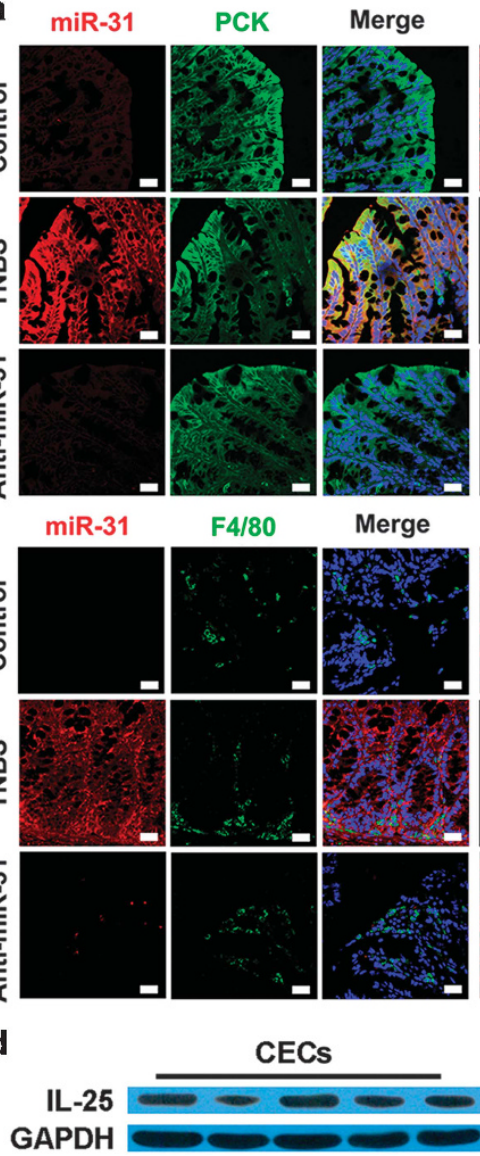

Merge

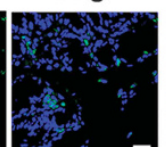

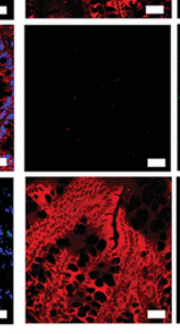

달:-
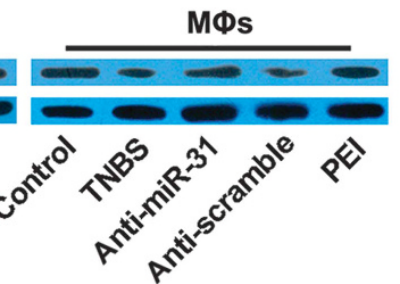

b
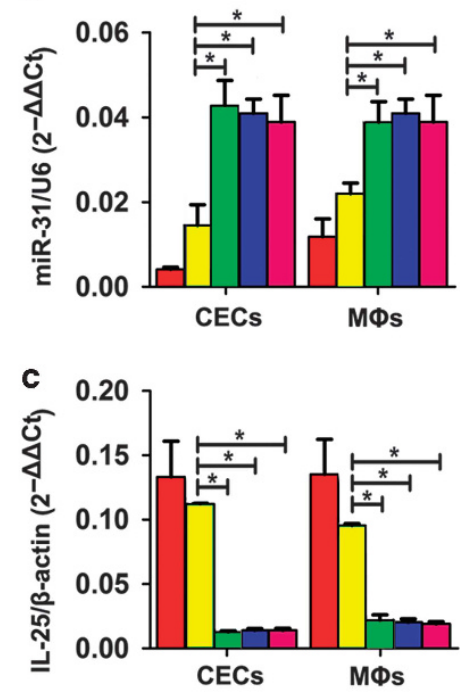

Control

PEI+anti-miR-31

TNBS

PEI+anti-scramble

PEI

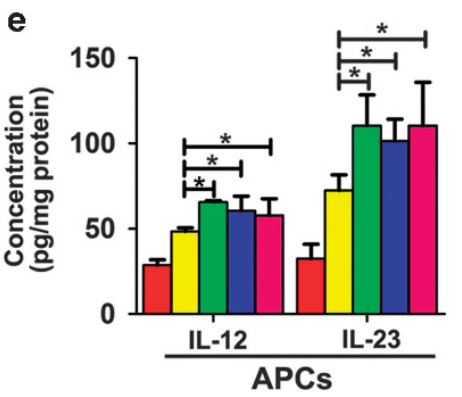

Figure 5 MiR-31/IL-25 affected the productions of IL-12 and IL-23 in 2,4,6-trinitrobenzene sulfonic acid (TNBS)-induced colitis after intracolonic administration of anti-miR-31. Immunofluorescence staining with colonic epithelial cell (CEC) marker (PCK) or M $\Phi$ marker (F4/80) and in situ hybridization for miR-31 was performed on TNBS-colitic colon sections after anti-miR-31 administration (red, miR-31; green, PCK or F4/80; blue, DPAI nuclear staining). Immunofluorescence staining to determine IL-25 localization was performed on TNBS-colitic colon sections following anti-miR-31 administration (red, IL-25; green, PCK or F4/80; blue, DPAI nuclear staining) (a). Scale bar, $25 \mu \mathrm{m}$. One representative image of five repeats was shown. MiR-31 level (b), IL-25 mRNA level (c), and IL-25 protein level (d) were examined in CECs or colonic MФs isolated from TNBS-colitic colons after anti-miR31 administration. Concentrations of IL-12 and IL-23 were measured in supernatants from antigen-presenting cells isolated from TNBS-colitic mice after anti-miR-31 administration and cultured for $12 \mathrm{~h}(\mathbf{e})$. The data are expressed as the mean \pm s.e.m. $n=5-7$ mice per group. ${ }^{\star} P<0.05$.

two experimental models (Supplementary Figures S5f and S6e). The administration of anti-miR-31 to mice significantly suppressed the expression of T-bet and ROR $\gamma t$ and decreased IFN- $\gamma$ and IL-17A levels (Supplementary Figures S5f,g and S6e,f). Meanwhile, flow cytometry analysis indicated that anti-miR-31 treatment decreased the proportion of colonic IFN- $\gamma^{+}$Th1 cells and IL-17A ${ }^{+}$Th17 cells (Figure 6d,h), suggesting that miR-31 might affect Th1/Th17-mediated colonic inflammation response through targeting IL-25 in two colitis models.

\section{Pre-miR-31 aggravated TNBS-induced colitis}

After colonic administration of pre-miR-31 to TNBS-induced mice with moderate colitis, miR-31 level increased and IL-25 production reduced in the colons (Supplementary Figure S7a,b). Images of the colons, body weight, DAI, and MPO activity of the mice demonstrated that the mice with moderate colitis developed more severe colitis after treatment with pre-miR-31 (Supplementary Figure S7c-f). Histological examinations confirmed these results (Supplementary Figure S7g,h). The expressions of IL-12 and IL-23 were enhanced and the levels of T-bet, ROR $\gamma$ t, IFN- $\gamma$, and IL-17A were elevated after the moderate colitis mice were treated with pre-miR-31 (Supplementary Figure 7i,j).

Therapeutic effects of anti-miR-31 on murine colitis were abolished by blocking IL-25 function or interfering with colonic IL-25 expression

As the administration of anti-miR-31 to colitis mice led to the upregulation of IL-25, we wondered whether the therapeutic effects of anti-miR-31 were IL-25 dependent. We found that the treatment of antibody against IL-25 could abolish the 

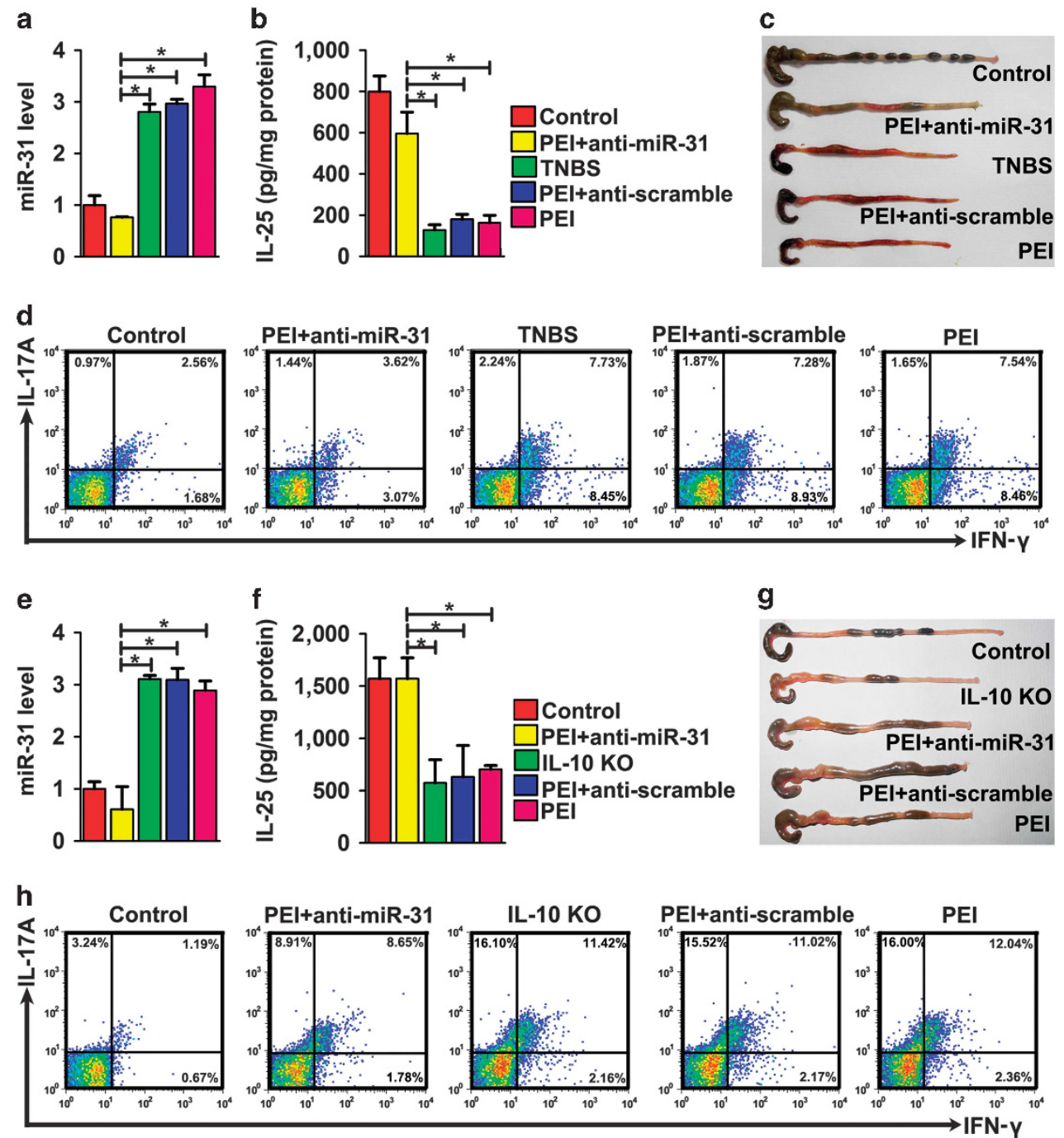

Figure 6 Anti-miR-31 treatment ameliorated 2,4,6-trinitrobenzene sulfonic acid (TNBS)-induced and IL-10 knockout (KO) colitis through targeting IL-25. MiR-31 level (a) and IL-25 production (b) were measured in TNBS-colitic colons after anti-miR-31 treatment. The effect of anti-miR-31 treatment on TNBS colitis was examined by colon images (c). Flow cytometry was used to analyze the ratio of Th1/Th17 cells in colon mucosal CD4 ${ }^{+} \mathrm{T}$ cells from TNBS colitis mice after anti-miR-31 administration (d). Moreover, miR-31 level (e) and IL-25 production (f) were examined in colons from IL-10 KO colitis mice after anti-miR-31 treatment. The therapeutic effect of anti-miR-31 on IL-10 KO colitis was determined by colon images (g). Flow cytometry was used to analyze the ratio of Th1/Th17 cells in colon mucosal CD4 ${ }^{+} \mathrm{T}$ cells from IL-10 KO colitis mice after anti-miR-31 treatment $(\mathbf{h})$. The data are expressed as the mean \pm s.e.m. $n=9-11$ mice per group. ${ }^{*} P<0.05$.

anti-inflammatory effects of anti-miR-31 on TNBS-induced colitis when compared with controls of rat IgG treatment or without any treatment (Figure $7 \mathbf{a}-\mathbf{c}$ and Supplementary Figure S8a-e). In addition, IL-25 shRNA lentiviruses, which significantly inhibited IL-25 expression in NIH-3T3 cells (data not shown), were administered to mice before TNBS treatment and anti-miR-31 injection. The administration of IL-25 shRNA lentiviruses significantly reduced colonic IL-25 expression compared with controls (Supplementary Figure S9b). The therapeutic effects of anti-miR-31 on TNBS-induced colitis were also abrogated by IL-25 shRNA lentiviruses (Supplementary Figure S9a-g).

Noteworthy, the treatment of IL-25 antibody or IL-25 shRNA lentivirus not only affected the inhibition of anti-miR31 on the secretion of IL-12 and IL-23 but also alleviated the suppression of anti-miR-31 on the expressions of T-bet, ROR $\gamma \mathrm{t}$, IFN- $\gamma$, and IL-17A (Figure 7e,f and Supplementary Figure S9h,i). Meanwhile, IL-25 mAb treatment recovered the proportion of colonic IFN- $\gamma^{+}$Th1 cells and IL-17A ${ }^{+}$ Th17 cells, which were downregulated by anti-miR-31 treatment (Figure 7d), which further indicated that the axis of miRNA-31/IL-25 could regulates Th1/Th17-mediated inflammation response in colitis.

\section{DISCUSSION}

Although clinical studies have indicated that the levels of IL-25 transcript and protein are significantly reduced in mucosal biopsy specimens taken from CD patients and UC patients, the biological function of IL-25, as an important regulatory factor during the colonic inflammation process, is 

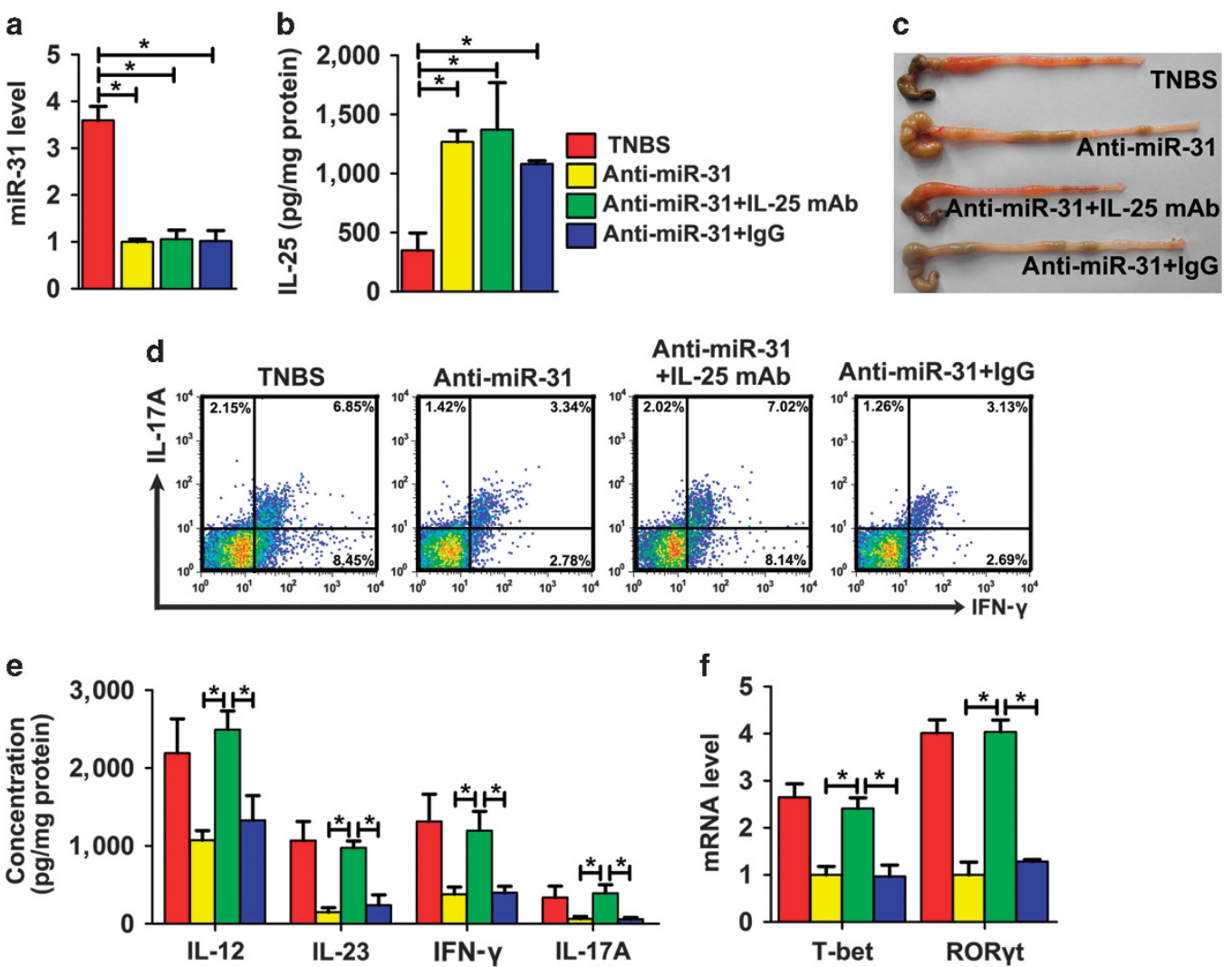

Figure 7 Effects of anti-miR-31 on 2,4,6-trinitrobenzene sulfonic acid (TNBS) colitis were abolished by treatment with antibody against IL-25. The level of miR-31 (a) and IL-25 production (b) in the colons of TNBS colitis was measured after co-treatment with IL-25 mAb and anti-miR-31. Clinical evolution was examined by colon images (c) after co-treatment with IL-25 mAb and anti-miR-31 in TNBS colitis. Flow cytometry was used to analyze the ratio of Th1/Th17 cells in colon mucosal CD4 ${ }^{+}$T cells from TNBS-colitic mice after IL-25 mAb and anti-miR-31 administration (d). The colonic inflammatory mediators (IL-12, IL-23, IFN- $\gamma$, and IL-17A) (e) and Th1 and Th17 transcription factors (T-bet and ROR $\gamma$ t) (f) were determined in TNBS colitis after co-treated with IL-25 mAb and anti-miR-31. The data are expressed as the mean \pm s.e.m. $n=9-11$ mice per group. ${ }^{*} P<0.05$.

not very clear. Previous studies indicated that the administration of exogenous IL-25 ameliorated variety of murine colitis models, including TNBS-induced colitis, peptidoglycan (PGN)-induced colitis, oxazolone-induced colitis, and DSS-induced colitis. ${ }^{15,27}$ Other discrepancy reports showed that the elevated colonic IL-25 level was observed in both oxazolone-induced colitis, a model mediated mainly by Th2-type inflammatory response, and TNBS-induced colonic tissue fibrosis caused by the repeated treatment with low dose of TNBS, which is also dependent on the development of IL-13 response. ${ }^{28,29}$ Although the studies on IL-25 in different colitis models were inconsistent, our present study indicated that the level of IL-25 was definitely deceased in two models (TNBS-induced acute colitis and IL-10 KO chronic colitis), which display human CD-like clinical and immunological features. ${ }^{30}$ From the previous reports, we found that the elevated colonic IL-25 level was observed in oxazolone-induced colitis (used to resemble UC patients) and colonic tissue fibrosis model, which is dependent on the development of IL-13 response. ${ }^{28}$ Briefly, IL-25 was decreased mainly in Th1/Th17 cells-mediated colitis and increased in experimental model when Th2 cells were involved. ${ }^{15,28}$
Moreover, the different pathogenesis of colitis and different types of targeting cells may lead to the distinct IL-25 biological functions during the colitis process. For example, the application of antibody against IL-25 considerably improved the histological characteristics of the oxazolone-induced colitis. $^{28}$ IL-25-deficient mice also showed resistance to DSS-induced colitis model. ${ }^{31}$ It is possible that CEC damage but not $\mathrm{T}$ cell response is the most important pathogenic factor during the initial process of DSS-induced acute colitis. In DSS-induced colitis, the enhanced IL-25 expression stimulated IL-6 secretion from CECs, which might further aggravate the epithelial cells dominated mucosal inflammation. ${ }^{31}$ As IL-17B could impair IL-25 signaling via competive binding to the IL-25 receptor (IL-17RB), IL-17B treatment might protect against colitis. Although there are discrepancy reports regarding the role of IL-25 in the development of Th2 cell or innate responsesmediated colonic inflammation, evidences clearly showed the anti-inflammatory role of IL-25 in Th1/Th17 dominated mucosal inflammation models including TNBS-induced acute colitis. ${ }^{14,15}$ Our finding that rIL-25 or IL-25 mAb alleviated or aggravated TNBS-induced colitis and IL-10 KO colitis via regulating mucosal Th1/Th17 responses were in correspondence with the previous literature. ${ }^{15}$ Therefore, investigating 
IL-25-expressed cell types, its biological function, and the detailed regulating mechanism especially in Th1/Th17mediated colonic inflammation is valuable for understanding the pathogenesis of $\mathrm{CD}$.

A previous report indicated that IL-25 transcripts were abundantly expressed in the intestinal epithelial cells of conventionally reared but not germ-free mice; whereas another report demonstrated that colonic IL-25 was primarily expressed by colonic $M \Phi s$ in the normal human gut. ${ }^{14,15}$ Our findings revealed that the IL-25 expression could be detected not only in CECs but also in colonic MФs in the colons of mice and humans. Moreover, the reduced IL-25 secretion could be observed in both two cell types in colitis mice and CD patients in this study. IL-25 is a key regulator of immune homeostasis on account of its ability to augment Th2 responses while suppressing Th1/Th17 cytokine responses. ${ }^{10}$ The decreased IL-25 production were unable to effectively suppress the production of IL-12 and IL-23 by APCs and impair the ongoing Th1/Th17 cell response in CD-like mucosal inflammation. ${ }^{10}$ Reports also indicated that IL-25 administration reduced the production of IL-12 or IL-23 by $\mathrm{CD} 14^{+}$cells or macrophages, and inhibited the production of Th1/Th17-associated cytokines in mice, hereby alleviating experimental colitis. ${ }^{14,15}$ It is consistent with our results that exogenous administration of rIL-25 suppressed mucosal Th1/Th17 responses and alleviated experimental colitis models, whereas injection of IL-25 mAb further enhanced Th1/Th17 responses and aggravated colitis. However, our data showed that exogenous IL-25 did not affect the Th2-associated cytokines (IL-4, IL-5, and IL-13) in TNBS-colitic or IL-10 KO colitic mice. Previous studies demonstrated that IL-17RB was not expressed by both human naive T, Th1 or Th17 cells, and mouse Th1 or Th17 cells, which suggest that the proliferation and differentiation of human Th1/Th17 cells and the proliferation of mouse Th1/Th17 cells cannot be directly affected by IL-25. ${ }^{32,33}$ Our finding further demonstrated that IL-25 could not directly affect the differentiation of mouse Th1/Th17 cells, but suppressed Th1/Th17 responses through an indirect way via regulating APCs function. In addition, we found that rIL-25 could improve colonic epithelial barrier function, which were consistent with previous reports. ${ }^{34,35}$ On the basis of the above mentioned evidences, we suppose that restoring colonic IL-25 expression may be beneficial to relief the clinical symbols of $\mathrm{CD}$ patients. Thus, seeking the potential regulatory mechanism of impaired IL-25 secretion during colonic inflammation may provide valuable clues for new drug development for $\mathrm{CD}$ treatment.

Recent studies based on cultured biopsies from CD patients revealed that IL- 25 expression was downregulated by TNF- $\alpha$, and commensal flora promoted IL-25 production in the intestine. ${ }^{14,16}$ However, the detailed molecular basis contributing to the reduced IL-25 level in colonic inflammatory context is not well understood. As an important post-transcriptional mechanism, miRNAs may contribute to these aberrantly expressed cytokines. Our results showed that among all the potential miRNAs targeting IL-25, only miR-31 was abnormally increased during mouse and human intestinal inflammation. Owing to the aberrantly expressed level in a variety of inflammation related diseases such as psoriasis, lupus, and colitis-associated neoplastic transformation, miR-31 may have a key role in organism immune. ${ }^{20,36-38}$ Cellular localization studies further demonstrated that miR-31 was primarily over-expressed in CECs and colonic MФs from the inflamed sites of TNBS colitis and CD patients. Although the reason for the elevated level of miR-31 in inflamed colonic cells remains unclear, it has been reported that TNF-stimulation could induce a significant upregulation of miR-31 in human intestinal epithelial LIM 1863 cells. ${ }^{9}$ In addition, IL-25 expression was significantly downregulated in CECs and colonic МФs. Therefore, we propose that the elevated miR-31 in CECs and colonic MФs, induced probably by proinflammatory cytokine stimulation, is an important molecular basis to control colonic IL-25 production and following Th1/Th17 cell responses in colitis models and human CD.

Moreover, we observed that the treatment with miR-31 inhibitors reduced the IL-12/IL-23 level in APCs, suppressed the Th1/Th17-mediated responses, and ameliorated colonic inflammation with concomitant elevation of colonic IL-25. Owing to the multiple targeting ability of miRNA, ${ }^{39}$ it is possible that miR-31 inhibitors may exert its protecting ability via regulating other genes rather than IL-25. A list of reported and predicted miR-31-target genes involved in immune diseases was summarized (Supplementary Tables S3 and S4). However, there is no converse relationship between miR-31 and targets of RhoA, Smad3, STK40, and Foxp3 in the colon of CD patients. ${ }^{34,40-42}$ The protein expression of Mapk11 in the colon of CD patients was below detection level. ${ }^{43}$ Other targets including Cd48, Il13ra1, Il1r1, Il12rb, and Mapk1 had no co-inverse relationship with miR-31 in TNBS colitis. ${ }^{44-46}$ As a recently identified protein, no report indicated the expression change of factor inhibit HIF-1 (FIH-1) in either experimental colitis models or $\mathrm{CD}$ patients. Our results observed the elevation of colonic FIH-1, but there was no converse relationship between miR-31 and FIH-1 in TNBS colitis (data not shown). Our experimental data further proved this speculation. In vitro luciferase assays indicated that IL-25 could be a direct target of miR-31. In vivo, intracolonic administration of anti-miR-31 upregulated colonic IL-25 expression and enhanced IL-25-mediated suppressive effect on Th1/Th17 cell-mediated inflammatory responses, which ameliorated symptoms in mouse colitis. Most importantly, we observed that both IL-25 blocking antibody and IL- 25 shRNA lentivirus could abolish the therapeutic effects of anti-miR-31 in TNBS colitis. From the aspects of target screen and the experimental data, it was most likely that miR-31 exerted its biological function via specially regulating IL-25 expression during the colitis process.

In conclusion, the signaling axis of miR-31/IL-25 was found to be able to regulate the colonic IL-12/23-mediated Th1/17 inflammatory responses in murine colitis. Restoring colonic IL-25 expression via intracolonic administration of miR-31 inhibitor represents a promising approach that may be valuable for $\mathrm{CD}$ treatment. 


\section{METHODS}

Mucosal sample collection, cell culture, and chemical reagents. Colonic mucosal biopsy specimens from normal and inflamed sites of six CD patients were obtained from Jinling Hospital (Supplementary Table S5). Ethical approval was obtained from the local ethics committee. The informed consents were also obtained from patients for experimentation. Caco-2 and NIH/3T3 cells (ATCC, Manassas, VA, USA) were cultured in Dulbecco's modified Eagle medium (Life Technology, Grand Island, NY, USA) containing 10\% fetal bovine serum (FBS) (Life Technology). THP-1 cells (ATCC) were cultured in Roswell Park Memorial Institute medium (RPMI)-1640 (Life Technology) containing 10\% FBS. All cells were incubated at $37^{\circ} \mathrm{C}$ in a humidified atmosphere of $5 \% \mathrm{CO}_{2}$. The primary CECs, lamina propria mononuclear cells (LPMCs), colonic MФs, and colonic SMCs were isolated and maintained as described in the Supplementary Experimental Procedures. Chemical reagents were purchased from Sigma (St Louis, MO, USA).

Establishment of experimental colitis models. All of the animals received care according to Chinese legal requirements. The study protocols were approved by the animal care and use committee of Nanjing University. Female BALB/c mice with 6-8 weeks of age were obtained from the Laboratory Animal Center of Nanjing University (Nanjing, China). TNBS colitis was induced as indicated previously. ${ }^{47}$ Briefly, $3 \mathrm{mg}$ of TNBS (Sigma) in $100 \mu \mathrm{l} 50 \%$ ethanol was slowly administered to lightly anesthetized $\mathrm{BALB} / \mathrm{c}$ mice through a catheter inserted $4 \mathrm{~cm}$ into the colonic lumen. The mice were then held in a vertical position for $30 \mathrm{~s}$. The control mice were treated with $100 \mu \mathrm{l}$ of $50 \%$ ethanol. The challenged mice were killed in different time-points (h $0,12,24,36,48,64,72,84,96,120,144$, and 168) to analyze the correlation between miR-31 and IL-25 during TNBS-induced colitis.

At 7-8 weeks of age, C3Bir.129P2 (B6)-Il10tm1Cgn/Lt (IL-10 KO) mice and $\mathrm{C} 3 \mathrm{H} / \mathrm{HeJ}$ mice used as controls were purchased from the Laboratory Animal Center of Nanjing University. After maintained under conventionally reared conditions for 12 weeks, the IL-10 KO mice appeared spontaneous colitis at week 20 and were killed.

To examine the role of $\mathrm{IL}-25$ in colitis, $\mathrm{BALB} / \mathrm{c}$ mice were intraperitoneally treated with $0.4 \mu \mathrm{g}$ rIL-25 (BioLegend, San Diego, CA, USA), $100 \mu \mathrm{g}$ IL-25 mAb (BioLegend), or control rat IgG (BioLegend) immediately after intracolonic administration of $2.5 \mathrm{mg}$ TNBS. Then, the mice were killed at 3 days after the induction of colitis. In addition, IL-10 KO mice were intraperitoneally treated with $0.4 \mu \mathrm{g}$ rIL-25, $100 \mu \mathrm{g}$ IL-25 mAb, or control rat IgG weekly from the ages of 12-20 weeks and the mice were finally killed at week 20 .

Flow cytometry analysis. For intracellular IFN- $\gamma$ and IL-17A staining, $\mathrm{CD} 4{ }^{+} \mathrm{T}$ cells sorted from LPMCs or in vitro differentiated $\mathrm{T}$ cells were stimulated with PMA $\left(50 \mathrm{ng} \mathrm{ml}^{-1}\right)$, ionomycin $\left(500 \mathrm{ng} \mathrm{ml}^{-1}\right)$ and brefeldin A $\left(10 \mu \mathrm{g} \mathrm{ml}^{-1}\right.$ ) (all from Sigma) for $4 \mathrm{~h}$. Then cell surface was stained with FITC-conjugated antibody against CD4 (BioLegend) and fixed in $1 \times$ intracellular fixation buffer (eBioscience, San Diego, CA, USA). Fixed cells were permeabilized with $1 \times$ permeabilization buffer (eBioscience) and followed by intracellular staining with PE-conjugated antibody against IFN- $\gamma$ (BioLegend) and allophycocyanin-conjugated antibody against IL-17A (BioLegend) allowing analysis by a flow cytometry analyzer (BD FACSCalibur, BD Biosciences).

Cell transfection. Precursor oligonucleotides (pre-miR-31), antisense oligonucleotides (anti-miR-31), and their scrambled non-coding RNAs (pre-scramble/anti-scramble) as controls were purchased from Ambion (Austin, TX, USA). Pre-scramble/anti-scramble was nonhomologous to any mouse or human genome sequence. The cells cultured in 6-well plates were transfected with the transfection mix containing 50 pmol of pre-miR-31 or 100 pmol of anti-miR-31 using Lipofectamine 2000 (Life Technology). The control experiments were performed in the same manner, except that mock-transfected cells were used. APCs isolated from colons of TNBS colitis were treated with supernatants collected from TNBS CECs $24 \mathrm{~h}$ after pre/anti-miR31 transfection, or exposed to the above collected supernatants supplemented with rIL-25 $\left(100 \mathrm{ng} \mathrm{ml}^{-1}\right)$ or IL-25 mAb $\left(2 \mu \mathrm{g} \mathrm{ml}^{-1}\right)$. The supernatants were collected for cytokines detection by ELISA and the cellular RNA was prepared for RNA studies $24 \mathrm{~h}$ after transfection. Cell lysate proteins were detected by western blotting $48 \mathrm{~h}$ after transfection. All transfections were replicated in triplicate.

Total RNA isolation and qRT-PCR. Total RNA was prepared using Trizol reagent (Life Technology) according to the manufacturer's instructions. RNA prepared from colonic T cells was used for T-bet and ROR $\gamma \mathrm{t}$ mRNA detection. To determine colonic miR-31 level and mRNA levels of IL-25, Claudin-1, ZO-1, and Occludin, RNA was prepared from the whole colon tissue. qRT-PCR was performed on an ABI 7300 Sequence Detection System (Applied Biosystems, Foster City, CA, USA) using EvaGreen Dye (Biotium, Hayward, CA, USA) For mRNA detection, $500 \mathrm{ng}$ of total RNA were used for complementary DNA synthesis (One Step SYBR PrimeScript RT-PCR Kit, Takara, Shiga, Japan), according to the manufacturer' instructions. The PCR conditions were as follows: $95^{\circ} \mathrm{C}$ for $10 \mathrm{~min}$, and then 40 cycles of amplification for $30 \mathrm{~s}$ at $95^{\circ} \mathrm{C}, 45 \mathrm{~s}$ at $60^{\circ} \mathrm{C}$, and $45 \mathrm{~s}$ at $72^{\circ} \mathrm{C}$. For miR-31 detection, $2 \mu \mathrm{g}$ of total RNA were used for first-strand DNA synthesis using AMV reverse transcriptase (Takara) and a stemloop RT-primer (Life Technology) under the following conditions: $16{ }^{\circ} \mathrm{C}$ for $30 \mathrm{~min}, 42^{\circ} \mathrm{C}$ for $30 \mathrm{~min}$, and $85^{\circ} \mathrm{C}$ for $5 \mathrm{~min}$. The conditions for the SYBR Green PCR were as follows: $95^{\circ} \mathrm{C}$ for $5 \mathrm{~min}, 95^{\circ} \mathrm{C}$ for $15 \mathrm{~s}$, and $60^{\circ} \mathrm{C}$ for $1 \mathrm{~min}$, for 40 cycles. The primers for qRT-PCR are shown in Supplementary Table S6. Sample and reference genes were analyzed in triplicates, and mRNA expression was normalized to $\beta$-actin, whereas miRNA expression was normalized to small nuclear RNA U6.

Treatment and evaluation of experimental colitis. The complexes of polyethyleneimine (PEI, $25 \mathrm{kDa}$, Sigma) and miR-31 precursor or inhibitor were prepared by mixing an aqueous solution of PEI $\left(4 \mathrm{mg} \mathrm{ml}^{-1}\right)$ with an equal volume of a solution of miR-31 precursor/ inhibitor $\left(2 \mathrm{mg} \mathrm{ml}^{-1}\right)$ and used for in vivo experiments as described previously. ${ }^{26}$ To determine the effect of anti-miR-31 on TNBS colitis, mice were treated with PEI/anti-miR-31, PEI/ anti-scramble, PEI alone, or saline $12 \mathrm{~h}$ after $3 \mathrm{mg}$ TNBS injection, at the dose of $5 \mathrm{mg}$ miRNA inhibitor/kg body weight. To examine the influence of pre-miR-31 on TNBS colitis, mild colitis was induced by administering $2 \mathrm{mg}$ TNBS to mice. Twelve hours later, the mice were given pre-miR-31 $\left(5 \mathrm{mg} \mathrm{kg}^{-1}\right)$.

To investigate whether the therapeutic effect of anti-miR-31 was based on IL-25, IL-25 mAb, or control rat IgG $(100 \mu \mathrm{g})$ was given intraperitoneally at the time of TNBS challenge. Twelve hours after $3 \mathrm{mg}$ TNBS induction, anti-miR-31/PEI $\left(5 \mathrm{mg} \mathrm{kg}^{-1}\right)$ was intracolonically injected. In addition, $10^{8}$ PFU of IL-25 shRNA lentiviruses, control lentivirus, or PBS $(150 \mu \mathrm{l})$ were intracolonically injected into mice 4 days before the $3 \mathrm{mg}$ TNBS treatment. Twelve hours after TNBS induction, anti-miR-31/PEI $\left(5 \mathrm{mg} \mathrm{kg}^{-1}\right)$ was intracolonically injected.

For IL-10 KO chronic colitis, anti-miR-31/PEI $\left(5 \mathrm{mg} \mathrm{kg}^{-1}\right)$ was administered to the mice weekly from the ages of 12-20 weeks. The level of SAA, a colitis marker for IL-10 KO mice, was monitored periodically by ELISA kit (R\&D, Minneapolis, MN, USA). Mice were killed at week 20.

The mice in both above models were observed daily. Usually, the TNBS challenged mice were killed at 3 days after the induction of colitis. The colons were excised for macroscopic observation, histopathological analysis, qRT-PCR assay, MPO activity measurements (Life Technology), and cytokine analysis by ELISA kits. For weight observation, the TNBS-induced colitis mice were killed 8 days after establishment of the model. The grades of intestinal inflammation in TNBS-induced colitis were assessed using DAI as previously described. ${ }^{48}$ For the histopathological assay, serial paraffin sections of the colon were stained with $\mathrm{HE}$, 
and histological scoring was performed according to standard protocols. $^{48}$

Statistics. The results are expressed as the means \pm standard error (mean \pm s.e.m.). The differences between groups were analyzed by the Mann-Whitney $U$ test and, if appropriate, by the Kruskal-Wallis ANOVA test. A value of $P<0.05$ was considered significant.

SUPPLEMENTARY MATERIAL is linked to the online version of the paper at http://www.nature.com/mi

\section{ACKNOWLEDGMENTS}

This work was supported by the National Basic Research Program of China (2012CB517603), the National High Technology Research and Development Program of China (2014AA020707), the National Natural Science Foundation of China $(31170751,31071232,31271013,31571458$, 31400671 , J1103512, and J1210026), and the Ph.D. Programs Foundation of the Ministry of Education of China (20130091110037).

\section{AUTHOR CONTRIBUTIONS}

T.S. performed the experiment and wrote the manuscript; Y.X., Y.F., Q.S., Z.M., and J.M. performed the experiment; J.C. and Z.H. designed the experiment and wrote the manuscript; and J.Z. designed the experiment.

\section{DISCLOSURE}

The authors declared no conflict of interest.

(c) 2017 Society for Mucosal Immunology

\section{REFERENCES}

1. Khor, B., Gardet, A. \& Xavier, R.J. Genetics and pathogenesis of inflammatory bowel disease. Nature 474, 307-317 (2011).

2. Kaser, A., Zeissig, S. \& Blumberg, R.S. Inflammatory bowel disease. Annu. Rev. Immunol. 28, 573-621 (2010).

3. Abraham, C. \& Cho, J.H. Inflammatory bowel disease. N. Engl. J. Med. 361, 2066-2078 (2009).

4. Todd, J.A. D'oh! genes and environment cause Crohn's disease. Cell 141, 1114-1116 (2010)

5. Papadakis, K.A. \& Targan, S.R. Role of cytokines in the pathogenesis of inflammatory bowel disease. Annu. Rev. Med. 51, 289-298 (2000).

6. Maloy, K.J. \& Powrie, F. Intestinal homeostasis and its breakdown in inflammatory bowel disease. Nature 474, 298-306 (2011).

7. Brand, S. Crohn's disease: Th1, Th17 or both? The change of a paradigm: new immunological and genetic insights implicate Th17 cells in the pathogenesis of Crohn's disease. Gut 58, 1152-1167 (2009).

8. Maloy, K.J. \& Kullberg, M.C. IL-23 and Th17 cytokines in intestinal homeostasis. Mucosal Immunol. 1, 339-349 (2008).

9. Pizarro, T.T. \& Cominelli, F. Cytokine therapy for Crohn's disease: advances in translational research. Annu. Rev. Med. 58, 433-444 (2007).

10. Monteleone, G., Pallone, F. \& Macdonald, T.T. Interleukin-25: a two-edged sword in the control of immune-inflammatory responses. Cytokine Growth Factor Rev. 21, 471-475 (2010).

11. Fort, M.M. et al. IL-25 induces IL-4, IL-5, and IL-13 and Th2-associated pathologies in vivo. Immunity 15, 985-995 (2001).

12. Owyang, A.M. et al. Interleukin 25 regulates type 2 cytokine-dependent immunity and limits chronic inflammation in the gastrointestinal tract. J. Exp. Med. 203, 843-849 (2006).

13. Kleinschek, M.A. et al. IL-25 regulates Th17 function in autoimmune inflammation. J. Exp. Med. 204, 161-170 (2007).

14. Zaph, C. et al. Commensal-dependent expression of IL-25 regulates the IL-23-IL-17 axis in the intestine. J. Exp. Med. 205, 2191-2198 (2008).

15. Caruso, R. et al. Interleukin-25 inhibits interleukin-12 production and Th1 cell-driven inflammation in the gut. Gastroenterology 136, 2270-2279 (2009).

16. Fina, D. et al. Interleukin-25 production is differently regulated by TNF-alpha and TGF-beta1 in the human gut. Mucosal Immunol. 4, 239-244 (2011).
17. Makeyev, E.V. \& Maniatis, T. Multilevel regulation of gene expression by microRNAs. Science 319, 1789-1790 (2008).

18. Carthew, R.W. \& Sontheimer, E.J. Origins and mechanisms of miRNAs and siRNAs. Cell 136, 642-655 (2009).

19. Wu, F. et al. Peripheral blood microRNAs distinguish active ulcerative colitis and Crohn's disease. Inflamm. Bowel. Dis. 17, 241-250 (2011).

20. Wu, F. et al. Identification of microRNAs associated with ileal and colonic Crohn's disease. Inflamm. Bowel Dis. 16, 1729-1738 (2010).

21. Fasseu, M. et al. Identification of restricted subsets of mature microRNA abnormally expressed in inactive colonic mucosa of patients with inflammatory bowel disease. PLoS One 5, e13160 (2010).

22. Brain, O. et al. The intracellular sensor NOD2 induces microRNA-29 expression in human dendritic cells to limit IL-23 release. Immunity $\mathbf{3 9}$, 521-536 (2013).

23. Huang, Z. et al. Dual TNF-alpha/lL-12p40 interference as a strategy to protect against colitis based on miR-16 precursors with macrophage targeting vectors. Mol. Ther. 23, 1611-1621 (2015).

24. Cheng, $X$. et al. miR-19b downregulates intestinal SocS3 to reduce intestinal inflammation in Crohn's disease. Sci. Rep. 5, 10397 (2015).

25. Wu, F. et al. MicroRNAs are differentially expressed in ulcerative colitis and alter expression of macrophage inflammatory peptide-2 alpha. Gastroenterology 135, 1624-1635.e1624 (2008).

26. Huang, Z. et al. miR-141 Regulates colonic leukocytic trafficking by targeting CXCL12beta during murine colitis and human Crohn's disease. Gut 63, 1247-1257 (2014).

27. McHenga, S.S., Wang, D., Li, C., Shan, F. \& Lu, C. Inhibitory effect of recombinant IL-25 on the development of dextran sulfate sodiuminduced experimental colitis in mice. Cell. Mol. Immunol. 5, 425-431 (2008).

28. Camelo, A. et al. Blocking IL-25 signalling protects against gut inflammation in a type-2 model of colitis by suppressing nuocyte and NKT derived IL-13. J. Gastroenterol. 47, 1198-1211 (2012).

29. Fichtner-Feigl, S. et al. Induction of IL-13 triggers TGF-1-dependent tissue fibrosis in chronic 2,4,6-trinitrobenzene sulfonic acid colitis. J. Immunol. 178, 5859-5870 (2007)

30. Wirtz, S. et al. Chemically induced mouse models of intestinal inflammation. Nat. Protoc. 2, 541-546 (2007).

31. Reynolds, J.M. et al. Interleukin-17B antagonizes interleukin-25-mediated mucosal inflammation. Immunity 42, 692-703 (2015).

32. Wang, Y.H. et al. IL-25 augments type 2 immune responses by enhancing the expansion and functions of TSLP-DC-activated Th2 memory cells. J. Exp. Med. 204, 1837-1847 (2007).

33. Angkasekwinai, P. et al. Interleukin 25 promotes the initiation of proallergic type 2 responses. J. Exp. Med. 204, 1509-1517 (2007).

34. Zhao, A. et al. Critical role of IL-25 in nematode infection-induced alterations in intestinal function. J. Immunol. 185, 6921-6929 (2010).

35. Buonomo, E.L., Cowardin, C.A., Wilson, M.G., Saleh, M.M., Pramoonjago, P. \& Petri, W.A. Microbiota-regulated IL-25 increases eosinophil number to provide protection during Clostridium difficile infection. Cell Rep. 16, 432-443 (2016).

36. $\mathrm{Xu}, \mathrm{N}$. et al. MicroRNA-31 is overexpressed in psoriasis and modulates inflammatory cytokine and chemokine production in keratinocytes via targeting serine/threonine kinase 40. J. Immunol. 190, 678-688 (2013).

37. Fan, W. et al. Identification of microRNA-31 as a novel regulator contributing to impaired interleukin-2 production in T cells from patients with systemic lupus erythematosus. Arthritis Rheum. 64, 3715-3725 (2012).

38. Olaru, A.V. et al. Dynamic changes in the expression of MicroRNA-31 during inflammatory bowel disease-associated neoplastic transformation. Inflamm. Bowel Dis. 17, 221-231 (2011).

39. Bartel, D.P. MicroRNAs: target recognition and regulatory functions. Cell 136, 215-233 (2009).

40. Horowitz, S. et al. Increased arginase activity and endothelial dysfunction in human inflammatory bowel disease. Am. J. Physiol. Gastrointest. Liver Physiol. 292, 1323-1336 (2007).

41. Di, Sabatino, A. et al. Transforming growth factor beta signalling and matrix metalloproteinases in the mucosa overlying Crohn's disease strictures. Gut 58, 777-789 (2009). 
42. Wang, Y., Liu, X.P., Zhao, Z.B., Chen, J.H. \& Yu, C.G. Expression of CD4 + forkhead box P3 (FOXP3) + regulatory T cells in inflammatory bowel disease. J. Dig. Dis. 12, 286-294 (2011).

43. Waetzig, G.H., Seegert, D., Rosenstiel, P., Nikolaus, S. \& Schreiber, S. p38 mitogen-activated protein kinase is activated and linked to TNF-alpha signaling in inflammatory bowel disease. J. Immunol. 168, 5342-5351 (2002).

44. Guzman, J. et al. ADOA3R as a therapeutic target in experimental colitis: proof by validated high-density oligonucleotide microarray analysis. Inflamm. Bowel Dis. 12, 766-789 (2006).

45. van der Pouw Kraan, T.C., Zwiers, A., Mulder, C.J., Kraal, G. \& Bouma, G. Acute experimental colitis and human chronic inflammatory diseases share expression of inflammation-related genes with conserved Ets2 binding sites. Inflamm. Bowel Dis. 15, 224-235 (2009).

46. Camacho-Barquero, L. et al. Curcumin, a Curcuma longa constituent, acts on MAPK p38 pathway modulating COX-2 and iNOS expression in chronic experimental colitis. Int. Immunopharmacol. 7, 333-342 (2007).

47. Neurath, M.F., Fuss, I., Kelsall, B.L., Stuber, E. \& Strober, W. Antibodies to interleukin 12 abrogate established experimental colitis in mice. J. Exp. Med. 182, 1281-1290 (1995).

48. Zuo, L. et al. Targeting delivery of anti-TNFalpha oligonucleotide into activated colonic macrophages protects against experimental colitis. Gut 59, 470-479 (2010). 